\title{
Analysis of hemodynamic responses to resistance exercise performed with different intensities and recovery intervals
}

\author{
Dihogo Gama de Matos ${ }^{1 *}$, Felipe José Aidar ${ }^{1}$, Mauro Lucio Mazini Filho ${ }^{1}$, \\ Rosimar da Silva Salgueiro ${ }^{2}$, Jordana Cristina de Oliveira ${ }^{2}$, Ingi P. Klain ${ }^{1}$, \\ Robert C. Hickner ${ }^{3}$, André Luis Carneiro ${ }^{1,4}$, Estélio Henrique Martin Dantas ${ }^{5}$ \\ ${ }^{1}$ Department of Sports Science, Exercise and Health of the Trás-os-Montes e Alto Douro University, Vila Real, Portugal; \\ *Corresponding Author: dihogogme@hotmail.com \\ ${ }^{2}$ University Center of Volta Redonda, Volta Redonda, Brazil \\ ${ }^{3}$ Departments of Kinesiology, and Physiology, Center for Health Disparities Research, East Carolina University, Greenville, USA \\ ${ }^{4}$ State University at Montes Claros (UNIMONTES), Montes Claros, Brazil \\ ${ }_{5}^{5}$ Doctoral Program in Nursing and Biosciences-PpgEnfBio, Federal University of State of Rio de Janeiro (UNIRIO), Rio de Janeiro, \\ Brazil
}

Received 19 October 2012; revised 18 November 2012; accepted 25 November 2012

\begin{abstract}
This aim of the present study was to analyze the hemodynamic responses during resistance exercise performed at different intensities and with different recovery intervals. This study was conducted on twenty-four apparently healthy male individuals $(25.50 \pm 3.72$ years and $76.50 \pm 4.50$ $\mathrm{kg}$ ) experienced in strength training. The volunteers performed a 1RM test to determine the training load for the study. Blood pressure and Rate Pressure Product were measured before and at the end of the exercise training. The only significant difference observed was in SBP during strength training at $70 \%$ intensity $(121.7 \pm$ 8.68, $p=0.039$ ), which was lower than SBP at the remaining intensities of $80 \%(126.3 \pm 7.11)$ and $90 \%(127.1 \pm 7.51)$. It was concluded that strength training performed at different intensities and recovery intervals did not significantly alter most variables, changing only the SBP due to the intensity employed.
\end{abstract}

Keywords: Strength Training (ST); Blood Pressure (BP); Heart Rate (HR); Rate Pressure Product (RPP)

\section{INTRODUCTION}

Strength training (ST) is usually practiced by people seeking benefits such as increased strength, change in body composition and health maintenance [1-3]. Strength training, when related to physical fitness, significantly reduces the risk of cardiovascular disease, and tends to cause autonomic and hemodynamic adaptations that influence the cardiovascular system to maintain cellular homeostasis in the face of increased metabolic demands $[4,5]$.

Exercise challenges the ability of the coronary arteries to provide enough blood to meet the demand of myocardial oxygen consumption. Heart rate (HR) and systolic blood pressure increase with exercise intensity $[6,7]$. Systolic blood pressure (SBP) is used to estimate the pressure exerted against the walls of the arteries during ventricular contraction and diastolic blood pressure (DBP) is used to estimate the pressure exerted against the artery walls when blood is not being forcibly ejected through vessel. Blood pressure (BP) alternates between the systolic level and the diastolic level of 120 to $80 \mathrm{mmHg}$ (in rest) [8].

Citing some studies of hypertensive patients, the American College of Sports Medicine Position Stand (ACSM) [9] states that physical activity is important in primary prevention and control of hypertension. The frequency, intensity, time and type of exercise (FITT) are specifically important factors in determining the physiological responses to exercise. Therefore, specific physical activities have been proposed because of their associations with better health and quality of life. However, such activities should meet certain safety criteria when they are prescribed [10].

Among the variables mentioned, yet seldom studied, is the length of periods of rest between sets and different types of exercises, which influences the total stress of training and total load that can be completed [11]. This 
variable is considered valuable when developing a program of strength training. Different periods of time between sets and exercises have been used in accordance with the objectives to be achieved.

The doubled-product (or rate pressure product, RPP), defined by the product of HR and SBP, is a variable correlated with myocardial oxygen consumption $\left(\mathrm{MVO}_{2}\right)$, and is therefore considered the most reliable indicator of the work of the heart during aerobic and anaerobic physical efforts [12]. The RPP is of great importance for the prescription and monitoring of these activities in healthy subjects or in those who have heart disease [12]. Little is known about the responses of HR and RPP during different work interval intensities and recovery interval durations despite the increased frequency of using strength training in hypertensive patients.

The aim of this study was therefore to analyze the response of blood pressure, heart rate and rate pressure product after strength training exercise using different intensities and recovery intervals.

\section{METODOLOGY}

\subsection{General Protocol}

The study consisted of twenty-four male individuals $(25.50 \pm 3.72$ years, $76.50 \pm 4.50 \mathrm{~kg})$ with a minimum experience of 12 months in ST, released for participation in the intervention by a physician. All participants responded negatively to the PAR-Q $[13,14]$. The volunteers were informed about the study, and all signed an authorization according to resolution no. 196/1996 of the National Health Council, for human experiments, in accordance with the ethical principles contained in the Declaration of Helsinki (1964, revised in 1975, 1983, 1989, 1996 and 2000), the "World Medical Association".

Participants were instructed not to consume caffeine or alcohol for the 24 hours prior to data collection and not to consume food for the 3 hours before the tests: this fact being conferred through interview after the tests. In addition, exclusion criteria were the use of ergogenic aids and medications that could affect cardiovascular responses, as well as SBP and DBP higher, than 139 and 89 $\mathrm{mmHg}$, respectively. These criteria were reconfirmed at each evaluation.

An interview (anamnesis) as well as anthropometric measurements of weight, height and skinfold were conducted [15]. There were two sessions for familiarization and two sessions training load determination, conducted with a minimum of 48 hours between sessions. The training load was established through the 1RM testing of Leg Press. The load determination was performed in two days: one day for testing and the second day for retesting to determine the reliability of the test. Figure 1 symbolizes the standardization of tests.

\subsection{Test of 1 Repetition Maximum (1RM)}

The 1RM tests were conducted twice after two familiarization sessions according to the protocol proposed by Brown et al. [16]. The subjects performed 3 - 5 min of light activities involving the muscle group tested, and after 1 min of light stretching, a warm-up of 8 repetitions at a perceived load of approximately 50\% 1RM, followed by 3 repetitions at a perceived load of approximately $70 \% 1 \mathrm{RM}$. After a five-min recovery period, the $1 \mathrm{RM}$ test was conducted by adding when necessary 0.4 to $5 \mathrm{~kg}$ to the load until the load could not be successfully lifted. The total number of attempts was 3 - 5 attempts. The maximum load that was raised in one motion was recorded as the 1RM load.

On the fifth day of the trial, after two familiarization sessions and two sessions to determine the maximum load, the SBP, DBP and HR were measured after the subject was seated for 10 minutes in a comfortable position in a quiet environment. After determining the load for the training, the experimental protocol was conducted on three additional days, not consecutive, with a minimum duration of 48 hours between sessions.

On the sixth day of trial, SBP, DBP and HR at rest and after exercise were again measured. Participants, after measuring variables at rest, performed 3 sets with 10 repetitions at $70 \%, 8$ repetitions at $80 \%$ and 6 repetitions at $90 \%$ of $1 \mathrm{RM}$ load in succession [17-20], with sixty second rest intervals. On the seventh and eighth day of assessments, training was repeated, but with intervals between sessions of, 60, 90, and 120 seconds, respectively. If at least one cardiovascular variable in relation to the value observed on the first day, the exercise was not performed and the participant was asked to revisit. Throughout the experiment we adopted a minimum rest of 48 hours between sessions.

Aiming to reduce the margin of error in the test we adopted the following strategies: standardized instructions were provided before testing, the subject was instructed to technical execution of the exercise, including

\begin{tabular}{|c|c|c|c|}
\hline $\begin{array}{l}1 \text { - day } \\
\text { Selection of } \\
\text { volunteers }\end{array}$ & $\begin{array}{l}2 \text { - day- } \\
\text { Interview and } \\
\text { anthropometric } \\
\text { measurements }\end{array}$ & $\begin{array}{l}3 \text { - day } \\
\text { Familiarization } \\
\text { of } 1 \mathrm{RM} \text { test }\end{array}$ & $\begin{array}{l}4 \text { - day } \\
\text { Retesting of } \\
\text { Familiarization } \\
\text { of 1RM test }\end{array}$ \\
\hline $\begin{array}{l}5 \text { - day } \\
\text { Familiarization } \\
\text { of training load }\end{array}$ & $\begin{array}{l}6 \text { - day } \\
\text { Retesting of } \\
\text { Familiarization } \\
\text { of training load }\end{array}$ & $\begin{array}{l}7 \text { - day } \\
\text { SBP, DBP } \\
\text { and HR were } \\
\text { measured }\end{array}$ & \\
\hline $\begin{array}{l}8 \text { - day } \\
3 \text { sets with } 10 \\
\text { repetitions at } \\
70 \%, 8 \text { repetitions } \\
\text { at } 80 \% \text { and } 6 \\
\text { repetitions at } 90 \% \\
\text { - } 60 \text { ' interval }\end{array}$ & $\begin{array}{l}9 \text { - day } \\
3 \text { sets with } 10 \\
\text { repetitions at } 70 \% \text {, } \\
8 \text { repetitions at } \\
80 \% \text { and } 6 \\
\text { repetitions at } 90 \% \\
-90 \text { ' interval }\end{array}$ & $\begin{array}{l}10 \text { - day } \\
3 \text { sets with } 10 \\
\text { repetitions at } \\
70 \%, 8 \text { repetitions } \\
\text { at } 80 \% \text { and } 6 \\
\text { repetitions at } 90 \% \\
-120 \text { ' interval }\end{array}$ & \\
\hline
\end{tabular}

Figure 1. Testing days. 
multiple familiarization and testing sessions to reduce possible effects of learning.

\subsection{Measurement of Blood Pressure}

$\mathrm{BP}$ and HR at rest, during exercise and during recovery were measured with an aneroid sphygmomanometer (Tycos ${ }^{\circledR}$, USA) and stethoscope (Littemann Quality ${ }^{\circledR}$, Germany) and with a heart rate monitor (Polar S725X, Polar Electro OY, Finland), respectively. For the measurement of HR and BP, we took into account the responses of peaks normally occurring during the last repetitions of a series [20]. Thus, data collection occurred at the end of the last repetition of the leg press exercise [20].

The BP measurement was performed in the left arm with the volunteers seated. The proper relationship between the cuff width and arm diameter arm was maintained for all subjects [21] (Kohlmann et al., 1999). Although not the gold standard for blood pressure measurements, our method provides continuous reading of SBP and DBP, and, in the case of the resistance exercise, the most suitable noninvasive method to measure blood pressure [11]. The blood pressure data were recorded in mmHg. Participants were asked to flex their arm at the elbow, with the level of their extended hand at the level of the thorax in order to prevent the data from being contaminated by gravitational action on the extended arm [21]. The subjects were also instructed not to contract or move the arm, hand and fingers where the stethoscope was attached, as well as to not perform the Valsalva maneuver. The measurement was performed at rest with the subject lying supine so there was no body movement between recordings.

\subsection{Statistics}

Descriptive analysis were performed, using measures of central tendency Data were presented as mean and standard deviation (mean $\pm \mathrm{SD}$ ). Normality of the data was checked using the Shapiro Wilk test. Two-factor ANOVA (Intensity and Interval) with Tukey post hoc was used to test for significance, with an alpha level of $p$ $<0.05$. Data analysis was performed using SPSS for Windows 15.0.

\section{RESULTS}

The HR, SBP, DBP and RPP in relation to intensity and rest interval duration are presented in Tables 1-4, respectively.

Systolic blood pressure was determined in 24 Participants at the end of a strength training protocol consisting of three sets, with 10 repetitions at $70 \%, 8$ repetitions at $80 \%$ and 6 repetitions at $90 \%$ of $1 \mathrm{RM}$ load with either 60,90 or 120 second rest intervals between sets.

Figure 2 shows the response of SBP in 24 participants during strength training at different intensities and interval.

Table 1. Heart rate (beats per minutes) at three intensities and three recovery intervals.

\begin{tabular}{ccccc}
\hline Intensity & Interval & $\mathrm{N}$ & Mean \pm SD & $\mathrm{p}$ \\
\hline $70 \%$ & & 24 & $117.33 \pm 18.52$ & 0.98 \\
$80 \%$ & & 24 & $116.42 \pm 18.25$ & \\
$90 \%$ & & 24 & $117.08 \pm 17.12$ & 0.95 \\
& $60^{\prime \prime}$ & 24 & $116.21 \pm 18.07$ & $117.92 \pm 16.68$ \\
& $90^{\prime \prime}$ & 24 & $116.71 \pm 19.06$ & \\
\hline
\end{tabular}

${ }^{*} \mathrm{p}<0.05$.

Table 2. Systolic blood pressure $(\mathrm{mmHg})$ at three intensities and three recovery intervals.

\begin{tabular}{cccccc}
\hline Intensity & Interval & $\mathrm{N}$ & Mean $\pm \mathrm{SD}$ & $\Delta \%$ & $\mathrm{p}$ \\
\hline $70 \%$ & & 24 & $121.7 \pm 8.68^{*}$ & 0.00 & 0.039 \\
$80 \%$ & 24 & $126.3 \pm 7.11$ & 3.78 & \\
$90 \%$ & 24 & $127.1 \pm 7.51$ & 4.43 & 0.074 \\
& & 24 & $127.1 \pm 8.07$ & 4.09 & 3.03 \\
\\
$60^{\prime \prime}$ & 24 & $125.8 \pm 7.17$ & 0.00 & \\
\hline
\end{tabular}

${ }^{*} \mathrm{p}<0.05$. 
Table 3. Diastolic blood pressure $(\mathrm{mmHg})$ at three intensities and three recovery intervals.

\begin{tabular}{ccccc}
\hline Intensity & Interval & $\mathrm{N}$ & Mean \pm SD & $\mathrm{p}$ \\
\hline $70 \%$ & & 24 & $85.83 \pm 18.86$ & 0.10 \\
$80 \%$ & & 24 & $98.33 \pm 11.29$ & \\
$90 \%$ & & 24 & $91.67 \pm 27.29$ & 0.69 \\
& $60^{\prime \prime}$ & 24 & $96.25 \pm 10.56$ & $95.42 \pm 11.79$ \\
\\
$90^{\prime \prime}$ & 24 & $84.17 \pm 31.06$ & \\
& $120^{\prime \prime}$ & 24 & & \\
\hline
\end{tabular}

${ }^{*} \mathrm{p}<0.05$.

Table 4. Rate pressure product $(\mathrm{mmHg} \times \mathrm{bpm})$ at three intensities and three recovery intervals.

\begin{tabular}{ccccc}
\hline Intensidade & Intervalo & $\mathrm{N}$ & Média \pm DP & $\mathrm{p}$ \\
\hline $70 \%$ & & 24 & $14283.75 \pm 2520.24$ & 0.70 \\
$80 \%$ & & 24 & $14700.42 \pm 2455.22$ & \\
$90 \%$ & & 24 & $14877.50 \pm 2304.00$ & 0.73 \\
& $60^{\prime \prime}$ & 24 & $14696.25 \pm 2037.33$ & $14862.08 \pm 2419.73$ \\
& $90^{\prime \prime}$ & 24 & $14303.33 \pm 2776.51$ & \\
\hline
\end{tabular}

${ }^{*} \mathrm{p}<0.05$.

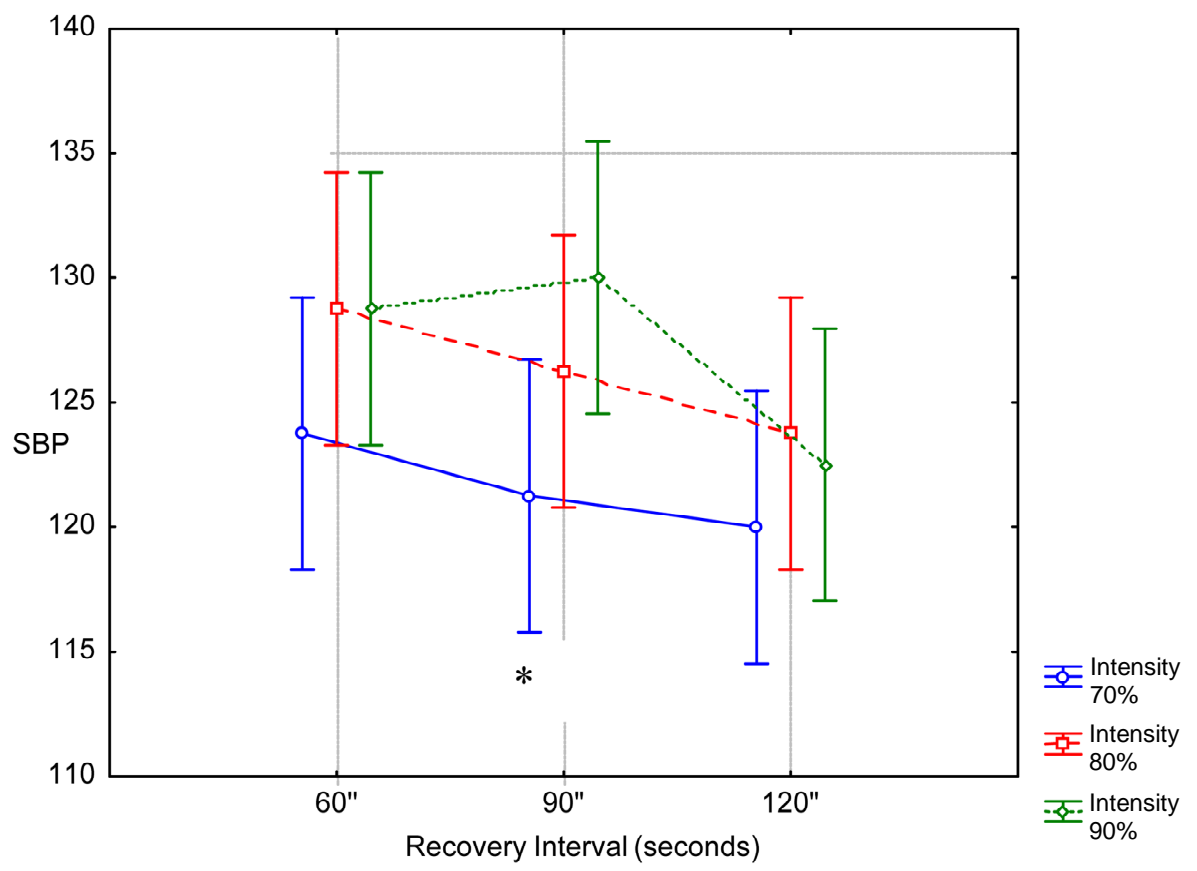

Figure 2. Systolic blood pressure during strength training comprised of different intensities and lengths of rest interval. ${ }^{*} \mathrm{p}<0.05$ for systolic blood pressure at $70 \%$ compared to $80 \%$ and $90 \%$.

No significant differences were observed in the following variables: HR - intensity $(\mathrm{p}=0.98)$, interval $(\mathrm{p}=$ $0.95)$ and intensity/interval $(p=1.00) ; \mathrm{SBP}$ - interval $(\mathrm{p}$ $=0.07)$, interval/intensity $(\mathrm{p}=0.82) ; \mathrm{DBP}$, intensity $(\mathrm{p}=$
$0.09)$, interval $(\mathrm{p}=0.06)$, intensity/interval $(\mathrm{p}=0.47)$ RPP, intensity $(p=0.70)$, interval $(p=0.73)$ interval/ intensity $(p=0.97)$. However, there was a significant difference in intensity (SBP: $p=0.03$ ). 


\section{DISCUSSION}

This aim of the present study was to analyze the hemodynamic responses during resistance exercise performed at different intensities and with different recovery intervals. The only significant difference observed was in SBP during strength training at $70 \%$ intensity $(121.7 \pm$ $8.68, \mathrm{p}=0.039)$, which was lower than SBP at the remaining intensities of $80 \%(126.3 \pm 7.11)$ and $90 \%$ $(127.1 \pm 7.51)$.

Although all individuals were able to complete the required number of sets in each series, shorter recovery time between them may have contributed to a higher physiological stress associated with that work intensity [22]. This could be attributed to two principal mechanisms: one of central and the other of peripheral origin [23]. The central mechanism involves the radiation of impulses from the motor cortex to central cardiovascular control. The peripheral mechanism consists of a reflex pathway that is yet to be fully described [24]. This mechanism is originated in the release of metabolites from muscles that are active (potassium and lactic acid, for example), increasing the osmolarity of interstitial fluid [25]. The release of these substances can activate chemoreceptor), which activates a feedback cardiovascular control center, increasing blood pressure [26,27]. In addition, the increased blood pressure can be influenced by the number of motor units required during exercise. In this case of increased motor unit recruitment, the adjustment would be perceived by mechanoreceptors which, like the chemoreceptor's, inform the cardiovascular control center of the need to modify the cardiovascular responses in relation to the strength and speed of movement [28].

The increase in SBP occurs during the ST due to considerable mechanical resistance to blood flow during muscle contraction. The ST increases blood flow, which results in increased peripheral resistance. This fact causes increased blood pressure, which corresponds to an increase in cardiovascular work, as evidenced by the increased rate pressure product [29-31].

There are few well-controlled studies of the changes in $\mathrm{BP}$ and HR associated with varying lengths of recovery interval between sets. The present study found no significant difference between protocols of different intensity $(p=0.98)$, and recovery interval $(p=0.95)$. It is possible that heart rate is associated with the exercise mode due to vascular occlusion being more pronounced in bilateral exercise. With vascular occlusion, venous return is reduced. HR should be increased to avoid compromising cardiac output [32]. However, there were no differences in recorded blood pressure or heart rate for the various series of exercises performed with different intensity or recovery periods.

In the experiment of Miranda et al. [10], the authors compared the heart hate, blood pressure and double product during bench press exercise while sitting or lying on the bench, with 10 repetitions performed at $65 \%$ of one repetition maximum (1RM). The authors did not identify significant differences in cardiovascular variables in their comparison of the performance in the seated or supine bench press. These results corroborate our findings in the present study.

Regarding RPP, no significant differences were found for intensity $(p=0.70)$ or recovery interval $(p=0.73)$. The fact that heart rate does not vary may have influenced the RPP results. Although the rate pressure product is not valid for estimating the myocardial oxygen consumption in activities of high intensity and short duration, it can be considered the best indicator of cardiac demand during resistance exercise $[33,34]$. However, the behavior of RPP depends not only on the intensity but also the type and duration of exercise. Until recently, there was a tendency to consider only aerobic activities safer for subjects with elevated risk of cardiac complications.

Exercises with weights were contraindicated for this population [34]. However, some studies show that the exercise double product with weights is usually lower than that observed in aerobic activities of moderate intensity $[10,35,36]$.

A limitation of this study is the measurement of BP by auscultation because of potential appraiser error. The intra-arterial catheterization, although more accurate in measuring BP, was not feasible for this study due to the invasive process and associated high risk for volunteers. Automated or ambulatory blood pressure monitoring (ABPM) is another reliable method that we could have used, although this system was not available for the current study.

\section{CONCLUSIONS}

Based on the results, we conclude that the resistance training conducted at different intensities and lengths of recovery does not result in measurable differences in diastolic blood pressure or rate pressure product. However, systolic blood pressure was increased with increased intensity.

It is therefore suggested that there be further studies analyzing different methods of strength training to understand the physiological parameters important to determining a safe and effective physical activity prescription.

\section{REFERENCES}

[1] Conley, M.S. and Rozenek, R. (2001) National strength and conditioning association position statement: Health aspects of resistance exercise and training. The Journal of Strength \& Conditioning, 23, 9-23.

[2] Bermudes, A.M., Vassallo, D.V. and Vasquez, E.C. (2003) 
Ambulatory blood pressure in normotensive individuals undergoing two single exercise sessions: Resistive and aerobic. Arquivos Brasileiros de Cardiologia, 2, 57-64.

[3] Dias, R.M.R, Cyrino, E.S., Salvador, E.P., Nakamura, F.Y., Pina, F.L.C. and Oliveira, A.R. (2005) Impact of eight weeks of weight training on muscle strength in men and women. Brazilian Journal of Sports Medicine, 11, 224-228.

[4] Pollock, M., Franklinm, B., Balady, G., Chaitman, B., Fleg, J. and Fletcher, B. (2000) Resistance exercise in individuals with and without cardiovascular disease. Circulation, 101, 828-833. doi:10.1161/01.CIR.101.7.828

[5] Polito, M.D., Simão, R., Senna, G.W. and Farinatti, P.T.V. (2003) Hypotensive effect of resistance exercise performed at different intensities and same workload. Brazilian Journal of Sports Medicine, 9, 69-73.

[6] Fisher, M.M. (2001) The effect of resistance exercise on recovery blood pressure in normotensive and borderline hypertensive women. Journal of Strength \& Conditioning Research, 15, 210-216.

[7] Whelton, S.P., Chin, A. and Xin, X. (2002) Effect of aerobic exercise on blood pressure: A meta-analysis of randomized, controlled trials. Annals of Internal Medicine, 136, 493-503.

[8] Polito, M.D. and Farinatti, P.T.V. (2003) Considerations for blood pressure measurement in exercises against resistance. Brazilian Journal of Sports Medicine, 1, 25-33.

[9] American College of Sports Medicine (2004) Exercise and hypertension. Medicine and Science in Sports and EXercise, 18, 533-553.

[10] Miranda, H., Simão, R., Lemos, A., Dantas, B.H.A., Baptista, L.A. and Novaes, J. (2005) Analysis of heart rate, blood pressure and double product in different body positions in resistance exercises. Brazilian Journal of Sports Medicine, 11, 25-32.

[11] Simão, R., Polito, M. and Monteiro, W. (2008) Effect of different recovery intervals in a program of strength training for individuals trained. Brazilian Journal of Sports Medicine, 14, 353-356.

[12] Gobel, F.L., Norstrom, L.A., Nelson, R.R., Jorgensen, C.R. and Wang, Y. (1999) The rate-pressure product as an index of myocardial oxygen consumption during exercise in patients with angina pectoris. Circulation, 57, 549-556. doi:10.1161/01.CIR.57.3.549

[13] Scott, T., Reading, J. and Shephard, R.J. (1992) Revision of the physical activity readiness questionnaire (PAR-Q). Canadian Journal of Sport Sciences, 17, 338-345.

[14] Tremblay, M.S, Wolfson, M. and Connor Gorber, S. (2007) Canadian health measures survey: Background, rationale and overview. Health Reports, 18, 7-20.

[15] Jackson, A.S. and Pollock, M. (1978) Generalized equations for predicting body density of men. British Journal of Nutrition, 40, 497-504. doi:10.1079/BJN19780152

[16] Brown, L.E. and Weir, J.P. (2001) ASEP Procedures recommendation I: Accurate assessment of muscular strength and power. Journal of Exercise Physiology Online, 4, 121.

[17] Delorme, T.L. and Watkins, A.L. (1948) Techniques of progressive resistance exercise. Archives of Physical Medicine, 29, 263-273.

[18] Delorme, T.L., Werst, F.E. and Shriber, W.J. (1950) Influence of progressive resistance exercises on knee function following femoral fractures. Journal of Bone and Joint Surgery, 32, 910-924.

[19] Delorme, T.L., Ferris, B.G. and Gallagher, J.R. (1952) Effect of progressive exercise on muscular contraction time. Archives of Physical Medicine, 33, 86-97.

[20] Fleck, S.J. and Kraemer, W.J. (2004) Designing resistance training programs. 3rd Edition, Human Kinetics, Champaign-Urbana.

[21] McLaughlin, V.M., Archer, S.A. and Badesch, D.B. (2009) ACCF/AHA clinical expert consensus document on hypertension: A report of the American college of cardiology foundation task force on expert consensus documents. Journal of the American College of Cardiology, 53, 1573-1619. doi:10.1016/j.jacc.2009.01.004

[22] Willardson, J.M. and Burkett, L.N. (2005) A comparison of 3 different rest intervals on the exercise volume completed during a workout. Journal of Strength and Conditioning Research, 19, 23-26.

[23] Woods, S., Bridge, T., Nelson, D., Risse, K. and Pincivero, D.M. (2004) The effects of rest interval length on ratings of perceived exertion during dynamic knee extension exercise. Journal of Strength and Conditioning Research, 18, 540-545.

[24] Carrington, C.A., Ubolsakka, C. and White, M.J. (2003) Interaction between muscle metaboreflex and mechanoreflex modulation of arterial baroreflex sensitivity in exercise. Journal of Applied Physiology, 95, 43-48.

[25] Wray, D.W., Uberoi, A., Lawrenson, L. and Richardson, R.S. (2005) Heterogeneous limb vascular responsiveness to shear stimuli during dynamic exercise in humans. Journal of Applied Physiology, 99, 81-86. doi:10.1152/japplphysiol.01285.2004

[26] Williams, M.A., Haskell, W.L. and Ades, P.A. (2007) Resistance exercise in individuals with and without cardiovascular disease: 2007 update: A scientific statement from the American heart association council on clinical cardiology and council on nutrition, physical activity, and metabolism. Circulation, 116, 572-584. doi:10.1161/CIRCULATIONAHA.107.185214

[27] Mitchell, J.H., Schibye, B., Payne, F.C. and Saltin, B. (1981) Response of arterial blood pressure to static exercise in relation to muscle mass, force development, and electromyographic activity. Circulation Research, 48, 170175.

[28] Prabhakar, N.R. and Peng, Y.J. (2004) Peripheral chemoreceptors in health and disease. Journal of Applied Physiology, 96, 359-366. doi:10.1152/japplphysiol.00809.2003

[29] Simão, R., Polito, M.D. and Lemos, A. (2003) Doubleproduct counter resistance exercises. Fit Perform Journal, 2, 279-84. doi:10.3900/fpj.2.5.279.p

[30] Leite, T. and Farinatti, P. (2003) Study of heart rate, blood pressure and double product in resistance exercises for various muscle groups similar. Brazilian Journal of Physiology Exercise, 2, 68-88. 
[31] Polito, M.D. and Farinatti, P.T.V. (2003) Considerations for blood pressure measurement in exercises against resistance. Brazilian Journal of Sports Medicine, 9, 2533.

[32] Farinatti, P.T.V. and Assis, B.F.C. (2000) Study of heart rate, blood pressure and double product in counter-resistance exercises and aerobic continuous. Brazilian Journal of Physical Activity and Health, 5, 5-16.

[33] American College of Sports Medicine (2000) Guidelines for exercising tests and prescription. 6th Edition, Lippincott Williams \& Wilkins, Baltimore.

[34] American College of Sports Medicine (2002) Position stand on progression models in resistance training for healthy adults. Medicine and Science in Sports and Exercise, 34, 364-380.

doi:10.1097/00005768-200202000-00027

[35] Miyai, N., Arita, M., Miyashita, K., Morioka, I., Shiraishi, T. and Nishio, I. (2002) Blood pressure response to heart rate during exercise test and risk of future hypertension. Hypertension, 39, 761-766. doi:10.1161/hy0302.105777

[36] O'Brien, E., Waeber, B., Parati, G., Staessen, J. and Myers, M.G. (2001) Blood pressure measuring devices: Recommendations of the European society of hypertension. British Medical Journal, 322, 531-536. doi:10.1136/bmj.322.7285.531

\section{ABBREVIATIONS}

ST: Strength Training

HR: Heart Rate

BP: Blood Pressure

SBP: Systolic Blood Pressure

DBP: Diastolic Blood Pressure
RPP: Rate Pressure Product

$\mathrm{MVO}_{2}$ : Myocardial Oxygen Consumption

1RM: Test of 1 Repetition Maximum

bpm: beats per minutes

ABPM: Ambulatory Blood Pressure Monitoring 